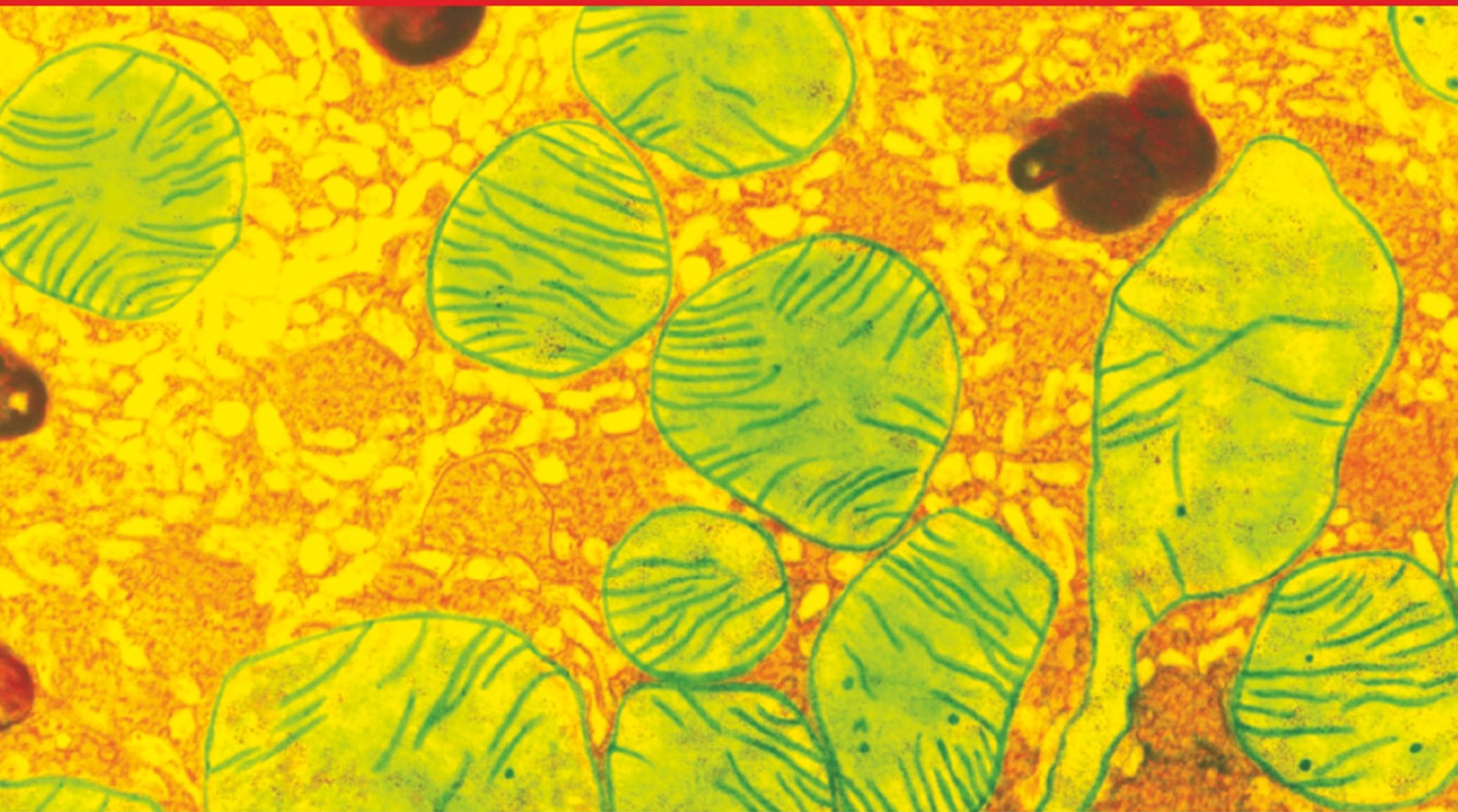

\title{
POWER GAMES
}

\section{There's a fight going on inside all our cells for each breath of air. Nick Lane sheds therapeutic light on the implications for cancer and degenerative diseases.}

$\square$ eventy-five years ago, Otto Warburg's star was at its zenith. The pioneering German biochemist delivered his Nobel address in December 1931. He described the ingenious experiments by which he had unmasked the enzyme responsible for the critical step of cell respiration, the process that turns the energy in chemical compounds into energy the cell can use. His work on respiration in the early 1930 s nearly earned him a second Nobel, ultimately denied him by Hitler. Then his star began sinking. His ideas on the importance of cell respiration in cancer led many to dismiss him as a crank. And the rise of molecular genetics in the 1960 s put such ideas into a far distant orbit.

Butnow, Warburg's star is rising again. A new generation of researchers is returning to his ideas about respiration in cancer cells. Recent findings suggest that the enzyme he identified, cytochrome oxidase, is a key player in a new understanding of how the cell's energy metabolism affects health and disease. And surprisingly they show that light has a profound effect on how the enzyme works - and could even be used to treat degenerative disease.

To extract energy from molecules, the cell first breaks down glucose into simpler mole- cules via a process called glycolysis. It then feeds these molecules into energy-producing structures called mitochondria, which strip electrons from them to produce energy with the help of oxygen. As Warburg showed, cytochrome oxidase governs the last reaction in this process.

Perhaps the most surprising aspect of the renaissance of Warburg's ideas is that the methods he used to make this discovery matter again. They exploit two chemical quirks: carbon monoxide (CO) can block respiration by binding to cytochrome oxidase in place of oxygen; and a flash of light can displace it, freeing up the site for oxygen to bind again.

"The finding that
the body could
poison one of
its own enzymes
was initially
shrugged off as
an imperfection."

\section{Self control}

By measuring oxygen consumption at different wavelengths of light, Warburg worked out that the enzyme belonged to a group of proteins that include haemoglobin and chlorophyll But for Warburg, the binding of CO to the enzyme was just an oddity he could put to good use. He had no inkling that biology might use the same trick.
Yet over the past decade, researchers have come to appreciate that cells often use $\mathrm{CO}$, and to an even greater extent $\mathrm{NO}$ (nitric oxide), to block respiration. Not only that, but light has striking counter-effects on cytochrome oxidase. And all these suitors to the enzyme turn out to be critical to our understanding not just of cancer, but practically all degenerative diseases.

Nitric oxide is emitted by nerve endings and can act on an enzyme called guanylate cyclase to relax blood vessels - the impotence drug Viagra manipulates this system. For a long time, scientists thought that guanylate cyclase was NO's only target. But in the mid-1990s, they found that the molecule can also bind to cytochrome oxidase and hinder respiration ${ }^{1}$. What's more, it could do so at levels found in the body's tissues.

The finding that the body could poison one of its own enzymes was initially shrugged off as an imperfection - an example of how evolution cobbles organisms together with no forethought. But a few years later, several groups reported that mitochondria harboured 
an enzyme that synthesizes NO (ref. 2). Why would cells go out of their way to cook up NO right next to the respiratory enzymes?

According to cell biologist Salvador Moncada of University College London, evolution really has crafted cytochrome oxidase to bind not only oxygen but also NO. "One effect of slowing respiration in some locations is to divert oxygen elsewhere in cells and tissues," he says. This prevents oxygen levels sinking dangerously low. Moncada, for example, has shown that NO blocks respiration in the cells lining blood vessels and that this helps to transfer oxygen into smooth muscle cells in these vessels. Fireflies use a similar trick to flash light (see 'Flashing the gauntlet').

But the consequences of blocking respiration go beyond diverting oxygen. Respiration,

"If we can modulate

this signalling, we

might be able to

influence the life

or death of cells in

many pathologies."

- Keshav Singh
Moncada argues, is not just about generating energy, it's about generating feedback that allows a cell to monitor and respond to its environment. Blocking respiration generates chemical signals, in the form of highly reactive molecules called free radicals. These are normally associated with cell damage, but now it seems theycan interact with the proteins that control gene activity and adapt cells to changing circumstances. "Free radicals had a bad reputation," says pathologist Victor DarleyUsmar, of the University of Alabama, Birmingham. "But we now see them as signals."

In the past few years, researchers have compiled a list of these proteins, or transcription factors, the activity of which depends, at least in part, on interactions with free radicals ${ }^{3}$. These include many proteins known to be linked to cellular life and death, such as P53, a protein that kills cells if they show signs of turning cancerous. "The question is," says Darley-Usmar, wis the whole system controlled?"

answer lies in the tens of thousands of Ine each mitochondrion. Organized into chains, they pass electrons extracted from broken-down glucose on to ach other. The end-point of the chain is cytochrome oxidase, which catalyses the final step of respiration, in which electrons and protons are transferred onto oxygen to form water. Energy released by this process is used to pump protons over a membrane, creating an electrical charge. The cell can then use this charge to power an enzyme that makes ATP, a molecule that fuels chemical reactions in the cell.

The cell can suppress the number of free radicals coming from these respiratory chains by allowing protons to leak back though the membrane without driving the synthesis of ATP, a process known as uncoupling ${ }^{4}$.

\section{Undercover radicals}

But if uncoupling doesn't bring free-radical leak under control, the signal may be amplified. “There's a cross-talk, known as the retrograde response, between the mitochondria and genes in the nucleus, which we're only just beginning to explore," says cell biologist Keshav Singh of the Roswell Park Cancer Institute in Buffalo, New York, who helped uncover this mechanism. "If we can modulate this signalling, we might be able to influence the life or death of
Gas station: by binding to the cytochrome oxidase enzyme (left), NO regulates respiration.

cells in many pathologies, even ageing."

In cancer cells, something goes awry with this pathway. Free radicals can trigger two steps in carcinogenesis, chromosomal instability and metastasis ${ }^{5}$, by hindering DNA repair and promoting the activity of genes involved in cancer spread. "We've found that damaged mitochondria, which overproduce free radicals, under-

\section{Flashing the gauntlet}

Firefly flashes have lit up the human imagination since antiquity. Light is produced by the action of the luciferase enzyme, which uses ATP to activate luciferin to a luciferyl adenylate intermediate. This in tum reacts spontaneously with oxygen to emit a photon, giving rise to bioluminescence.

The sequence of flashes, which often beam complex messages to potential mates, clearly requires exquisite nervous control. But no nervecells connect directly to the 'lantern' cells that produce the light, suggesting that some kind of intermediary is needed to ferry messages from the nerve endings.
Barry Trimmer and his colleagues at Tufts University in Medford, Mas sachusetts, have shown that this is likely to be NO (ref. 12) and suggestit works by binding to cytochrome oxidase ${ }^{B}$. To do so, it exploits a neat feature of the firefly's breathing system.

Insects breathe through tubules, called trachea, that connect the outside world to cells in the body via a network of increasingly fine branches. The ends of these, called tracheoles, abut cells and allow oxygen to diffuse outwards. In fireflies, when oxygen passes from tracheoles into the lantern cells, itmust run the 'gauntlet' - a

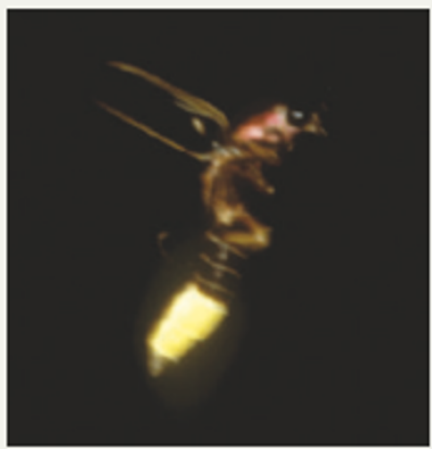

dense ring of energy-producing structures called mitochondria in the periphery of the lantern cells. Normally, the oxygen would bind to cytochrome oxidase in the mitochondria and beused in respiration. But when $N O$ is released from the nerveendings, it binds to cytochrome oxidase, blocking respiration and diverting oxygen through the gauntlet into the lantern cell.

There, oxygen reacts with the luciferyl intermediate to produce a flash of light. The glory is that the flash switches itself off. Light dissociates NO from cytochrome oxidase, allowing oxygen to bind again. Now the mitochondria consume oxygen oncemore, allowing the luciferylintermediate to build up until another wave of $\mathrm{NO}$ arrives.

N.L 


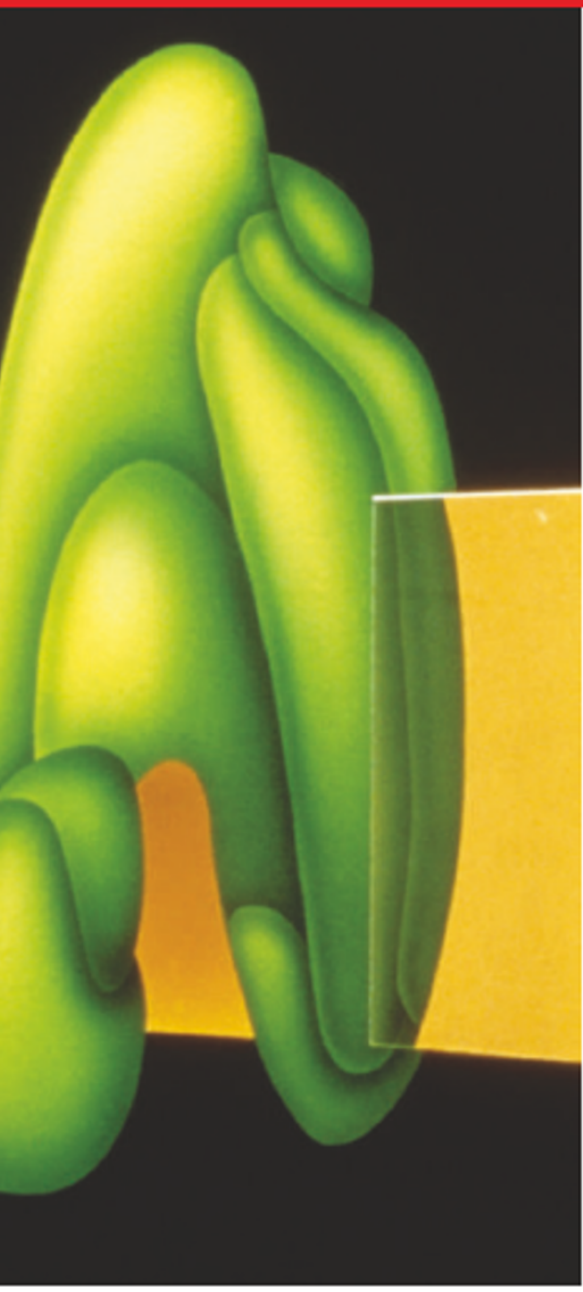

mine the integrity of the nuclear genome in otherwise normal cells," says Singh.

Ultimately, however, the most intractable problem with cancer cells is their failure to undergo apoptosis (cell suicide).There are echoes of Warburg here, for he argued that cancer cells revert to a primitive type that has little or no need for oxygen or mitochondria, depending instead on anaerobic forms of energy generation such as glycolysis. Whether cells really 'revert' is a moot point, but Moncada has shown that cells' ability to switch to glycolysis is critical to their survival ${ }^{6}$.

Moncada's findings suggest that cells that can do without mitochondria - many stem cells, for example, which have been implicated in cancer - can have their resistance to apoptosis stiffened by NO binding to cytochrome oxidase, making cancer more likely. At the same time, cells that depend on mitochondria for energy, such as neurones, may be pushed to apoptosis by NO binding, making degenerative disease more likely.

Any solution to excessive NO binding might lower the risk of both cancer and degenerative diseases, as it would make apoptosis more likely in cancer cells and less likely in normal cells. And the second essential feature of Warburg's experiments - light - might do just that.

Light has long been known to promote wound healing, but the detailed molecular mechanisms have only recently been studied. Light's effects are more than skin deep: at long wavelengths, in the near infrared (NIR) spectrum, photons may penetrate several centimetres into the body.

And according to photobiologist Tïina Karu, at the Russian Academy of Sciences in Moscow, NIR rays in exactly this range modulate cell respiration and the signals it generates.

\section{Light works}

Karu's group has explored a number of changes taking place in cultured cells in response to NIR rays. The immediate effect is an energy 'buzz', in which ATP levels are stoked up and the electrical charge across the mitochondrial membrane is strengthened. A few hours later, the activity of as many as 110 genes shifts in concert $t^{3}$. These genes orchestrate a prolonged rise in mitochondrial energy production, as well as stress resistance. They also prompt cells to cling more strongly to their surroundings, an important factor in wound healings.

Exactly how NIR light interacts with the enzyme to bring about these changes is unclear, and difficult to measure.

One idea is that phototherapy might work by dissociating NO from the enzyme, so reversing the signalling consequences of excessive NO binding.

"We have shown that light can indeed reverse the inhibition caused by NO binding to cytochrome oxidase, both in isolated mitochondria and in whole cells," says biochemist Guy Brown, at the University of Cambridge, UK. "And what's more, we found that Healing glow? Lightcan prevent light can protect cells against cells from killing themselves. NO-induced cell death.

He has a reservation, however. These experiments used light in the visible spectrum, with wavelengths from 600 to $630 \mathrm{~nm}$. Although Brown acknowledges that cytochrome oxidase absorbs NIR photons from 700 to $900 \mathrm{~nm}$, he points out that the absorption takes place in part of the enzyme not involved in NO binding. NIR also seems to have effects on cytochrome oxidase in conditions where NO is unlikely to be present. Presumablythen, says Brown, NIR must also have a direct effect on the enzyme.

Regardless of the exact mechanism, the effect is to relieve a blockade of the enzyme, whether by NO or any other molecules. According to toxicologist Janis Eells, at the University of Wisconsin, Milwaukee, such relief lowers the likelihood of apoptosis in many conditions.

Eells and her colleagues found that NIR phototherapy counters methanol poisoning, which injures the retina and optic nerve, often causing blindness'. The toxic metabolite is formic acid, which inhibits cytochrome oxidase. "In a rat model, NIR phototherapy is able to restore virtually normal retinal function, at least as judged by the electroretinogram," says Eells.

And neurobiologist Margaret Wong-Riley and her colleagues at the Medical College of Wisconsin in Milwaukee have shown that NIR phototherapy can also oppose the effects of cyanide on cell cultures ${ }^{10}$. Cyanide poisons by binding to cytochrome oxidase. Wong-Riley's team showed that phototherapy could halve the rate of apoptosis in cultured neurones, even when given before cyanide treatment.

But can NIR phototherapy relieve not just acute toxicity, but more chronic inflammatory conditions? The signs augur well. Eells and her colleagues have shown that NIR phototherapy could cut the rate of apoptosis by $50 \%$ in a rat model of retinitis pigmentosa, in which photoreceptors die by apoptosis during postnatal development causing retinal degeneration and blindness ${ }^{11}$. The use of NIR phototherapy on other conditions in which cells die by apoptosis, including acute ischaemic stroke and myocardial infarction, has also shown promise in animal models, and should soon enter clinical trials.

So will it work? "The trouble is that if you enthuse about light as a therapy, dinicians and even researchers tend to back away," says Eells. "Perhaps if the physical interactions of photons with cytochrome oxidase and NO are better known, people will begin to appreciate the huge potential benefits of this technique."

Nick Lane is a science writer based in London.

1. Moncada, S. \& Erusal imsky, J.D. Nature Rev. Mal. Cell Bial. 3, 214-220 (2002).

2. Brown G C. Biochim. Blophys. Acta 1504, 46-57(2001).

3 Darley-Usmar, V. Free Rad Bial. Med 37, 753-754 (2004).

4. Brand M. D. Biochem. Soc Trans 33, 897-904 (2005).

5. Singh K K etal. Gene 354, 140-146 (2005).

6. Moncada, S. \& Bolahlos, L.P.J. Neurochem. 97, 1676-1689 (2006).

7. Zhang, Y.et a. J. Invest. Dermatal. 120, 849-857 (2003)

8. Karu T. L, Pyatibrat, L. V.\& Afanasyeva, N. L Photochem. Photabiol. 80, 366-372(2004).

9. Eells, L T. etal Mitochandrian 4,559-567(2004).

10. Wong-Rilex, M. T.et al. J. Bial. Chem. 280, 4761-4771 (2005).

11. Eells, L. T. et al Near Infared Light Theapy for Retinit's Plgmentosa (ARVO, 2006).

12. Trimmer, B. A. et al. Sclence 292, 2486-2488 (2001).

13. Aprille, I R, Lagace, C. L, Modica-Napolitano, J.\& Trimmer B. A. Integr. Coma Bial. 44,213-219 (2004) 